\title{
RELACIÓN ENTRE LA RESILIENCIA Y LA SATISFACCIÓN CON LA VIDA EN UNA MUESTRA DE MUJERES CON CÁNCER DE MAMA
}

\author{
Antonio Zayas \\ Emotional Intelligence Laboratory \\ University of Cádiz (Cádiz, Spain) \\ Research Institute for Sustainable Social Development \\ (INDESS, Jerez de la Frontera, Cádiz, Spain) \\ antonio.zayas@uca.es \\ Rocío Gómez-Molinero \\ Emotional Intelligence Laboratory \\ University of Cádiz (Cádiz, Spain) \\ Rocío Guil \\ Emotional Intelligence Laboratory, \\ University of Cádiz (Cádiz, Spain) \\ Research Institute for Sustainable Social Development \\ (INDESS, Jerez de la Frontera, Cádiz, Spain)

\section{Paloma Gil-Olarte} \\ Emotional Intelligence Laboratory \\ University of Cádiz (Cádiz, Spain) \\ Research Institute for Sustainable Social Development \\ (INDESS, Jerez de la Frontera, Cádiz, Spain) \\ Encarna Jiménez Orozco \\ Oncology Unit of General Hospital of Jerez de la Frontera \\ (Jerez de la Frontera, Cádiz, Spain)
}

Fecha de Recepción: 14 Marzo 2018

Fecha de Admisión: 10 Abril 2018

\section{RESUMEN}

La Sociedad Española de Oncología Médica (SEOM, 2017) señala que el cáncer de mama es la neoplasia más frecuente en mujeres españolas. En las últimas décadas ha aumentado el número de estudios desde una óptica salutogénica. Así, la resiliencia, enmarcada dentro de la psicología positiva, es la capacidad de las personas para resistir y sobreponerse a situaciones traumáticas, (Águila, 2000). De acuerdo con la literatura, existe una relación positiva entre la resiliencia y el bienestar psicológico en mujeres con cáncer de mama (Guil et al., 2016). Por lo tanto, cabe esperar que la satisfacción con la vida guarde relación con la resiliencia, al tratarse de un concepto más amplio y 
con mayor estabilidad que el bienestar psicológico. Por tanto, nuestro objetivo es analizar la relación existente entre la resiliencia, en todas sus dimensiones, y la satisfacción con la vida, así como analizar la capacidad predictiva que tiene una sobre otra. La muestra estuvo conformada por 30 mujeres con cáncer de mama, con una media de edad de 47,47 años (SD=6.356) atendidas en el Hospital de Jerez en el momento de la evaluación. Se utilizó la Escala de Resiliencia de Wagnild y Young, (1993) y la Escala de Satisfacción con la Vida de Diener, Emmons, Larsen y Griffin. Los resultados muestran correlaciones positivas estadísticamente significativas entre la resiliencia, concretamente en sus dimensiones "Ecuanimidad", "Confianza en sí mismo", "Perseverancia" y la Satisfacción con la Vida. En los análisis de regresión, la resiliencia no ha mostrado capacidad predictiva significativa sobre la Satisfacción con la Vida. A pesar de haberse encontrado relación entre ambas variables, así como conocerse la dirección positiva de dicha relación, no se puede concluir que la resiliencia sea un predictor de la satisfacción con la vida en esta muestra.

Palabras claves: resiliencia; satisfacción con la vida; psicología positiva; cáncer de mama

\section{ABSTRACT}

Relationship between resilience and life satisfaction in a sample of breast cancer women.

The Spanish Society of Medical Oncology (SEOM, 2017) indicates that breast cancer is the most frequent neoplasia in Spanish women. In the last decades, there has been an increased number of studies from a salutogenic perspective. Thus, resilience, a construct framed within the positive psychology field, has been defined as the ability to resist and overcome traumatic situations, (Águila, 2000). According to the literature, there is a positive relationship between resilience and psychological well-being, in breast cancer women (Guil et al., 2016). Therefore, life satisfaction is expected to relate to resilience, since it is a broader concept with greater stability than psychological wellbeing. Therefore, the aim of the study is to analyze the relationship between resilience, including all its dimensions, and life satisfaction, as well as to examine the predictive ability that one has over another. The sample consisted of 30 breast cancer women, with a mean age of 47.47 years (SD = $6,356)$ attended at the Hospital of Jerez at the time of the evaluation. The Wagnild and Young Resilience Scale (1993) and the Life Satisfaction Scale of Diener, Emmons, Larsen, and Griffin were administered. The results show statistically significant positive correlations between resilience, specifically the dimensions "Equanimity", "Confidence in itself", "Perseverance", and Life Satisfaction. In regression analyzes, resilience has not shown significant predictive ability in Life Satisfaction. Despite the positive relationship between both variables, we cannot conclude that resilience is a predictor of life satisfaction in within this sample.

Keywords: resilience; life satisfaction; positive psychology; breast cancer

\section{ANTECEDENTES}

El cáncer es la enfermedad que más número de muertes causa en el siglo XXI, constituyendo un gran problema de salud pública (Guil, Zayas, Gil-Olarte, Guerrero, González \& Mestre, 2016). Siguiendo a estos autores, el hecho de padecer esta enfermedad influye de manera importante en múltiples aspectos de la vida del ser humano, como es el trabajo, las relaciones interpersonales 0 hábitos diarios, afectando claramente al bienestar físico, bienestar psicológico y la satisfacción con la vida de estos pacientes.

Concretamente el cáncer de mama representa la patología maligna más común en mujeres de los países occidentales (Bernardi et al., 2008; Von Humboldt, Carneiro \& Leal 2017). En España, la Sociedad Española de Oncología Médica (SEOM, 2016), señala que la neoplasia mamaria es el tipo de cáncer más común en las mujeres. Cuando se hace referencia a esta enfermedad, se entiende 
como el crecimiento maligno de las células epiteliales que rodean los conductos de la mama (Tescari, 2012).

El padecimiento de cáncer de mama conlleva grandes desajustes psicológicos y emocionales debido, entre otras cosas, a su carácter crónico, la incertidumbre que sobre su pronóstico sienten las mujeres que lo padecen, los efectos secundarios de los tratamientos médicos usados para combatirlo y el significado o representación social de la propia palabra cáncer (Alonso \& Bastos, 2011; Guil, Zayas, Gil-Olarte, Guerrero \& Mestre, 2017).

El estudio del impacto psicológico que el cáncer de mama tiene sobre las mujeres ha sido enfocado durante años desde un punto de vista patogénico. Son numerosos los autores que han investigado sobre los aspectos que influyen en el malestar emocional derivado del cáncer de mama, 0 sobre la eficacia de las intervenciones dirigidas a paliar los trastornos psicopatológicos comórbidos a la enfermedad, como es la ansiedad o la depresión (Zayas \& Guil, 2018). Sin embargo, más recientemente y con la aparición en el campo científico de la Psicología Positiva, está empezando a incrementar el interés por el estudio de aquellas variables psicosociales y emocionales que ejercen un papel importante en la prevención de trastornos psicopatológicos derivados del diagnóstico de cáncer de mama.

Barreto \& Soler (2008) enumeran diferentes factores protectores de la aparición de cuadros psicopatológicos derivados del padecimiento de cáncer de mama, entre los que se encuentra el hecho de que estas mujeres cuenten con fuentes internas de resiliencia.

Se podría definir la resiliencia como la capacidad que poseen las personas para resistir, sobreponerse y salir delante de manera exitosa después de haber pasado por dificultades 0 acontecimientos traumáticos (Aguila, 2000). Otros autores como Wagnild \& Young (1993) afirman que la resiliencia es una característica de personalidad que tiene un efecto moderador en el impacto negativo que el estrés tiene en la persona, fomentando la adaptación a dicha situación estresante. Por lo que cabe esperar que la resiliencia fomente la salud mental y la satisfacción con la vida, a pesar de experimentarse una situación traumática como es el diagnostico de cáncer de mama (Zayas \& Guil, 2018).

La mayoría de estudios científicos llevados a cabo concluyen que en las mujeres que padecen cáncer de mama, el ajuste psicológico es mucho más adecuado cuando éstas encuentran sentido a la experiencia (Bellizzi \& Blank, 2006; Scrignaro, Barni \& Magrin, 2010), tras los tratamientos tienen menos secuelas emocionales negativas (Aspinwall \& MacNamara, 2005; Aydin, 2008; Bozo et al., 2009; Salsman, Segerstrom, Brechting, Carlson \& Andykowski, 2009), se cuidan más durante el tratamiento, y una vez finalizado llevan una vida más saludable y un mayor compromiso con la salud (Vázquez \& Castilla, 2007). Es decir, que las mujeres que presentan mayores niveles de resiliencia aprovechan los aspectos positivos que pueden extraer de la enfermedad, lo cual garantizará mayor nivel de bienestar y satisfacción con la vida. Otros autores como García-Serrablo, Acevedo \& Ortiz (2011) indican que las mujeres con cáncer de mama han llegado a tener una percepción de su enfermedad de forma positiva, encontrándole beneficios a la misma, después de haber pasado por el sufrimiento derivado de los tratamientos médicos y la incertidumbre sobre el pronóstico, lo cual es indicativo de que el hecho de pasar por la enfermedad puede fortalecer a las mujeres. En otro estudio llevado a cabo por Crespo \& Rivera (2012) hallaron que mujeres con cáncer de mama mencionaban haber salido fortalecidas tras la enfermedad, pasaron a tener más fe, sentirse más capaces de solucionar problemas, poder relacionarse mejor con las personas que le rodean y tener menor preocupación sobre la enfermedad, pudiéndose inferir que estas mujeres presentan elevados niveles de resiliencia y por ende, mayor nivel de bienestar y satisfacción con la vida.

Cuando se hace referencia al bienestar psicológico, es importante hacer una distinción entre los 
elementos cognitivos y los emocionales. El estado emocional está más centrado en la experiencia a corto plazo, siendo la satisfacción con la vida una perspectiva más amplia en el tiempo y de mayor estabilidad (Vázquez, Duque \& Hervás, 2013). La satisfacción con la vida corresponde al componente de bienestar subjetivo y se define como la evaluación general que una persona realiza sobre su propia vida (Atienza, Pons, Balaguer \& García-Merita, 2000; Diener, Scollon \& Lucas, 2009; VeraVillarroel, Urzúa, Pavez, Celis-Atenas \& Silva, 2012).

Por lo que teniendo en cuenta todo lo anteriormente expuesto, se infiere que las mujeres que han sido diagnosticadas de cáncer de mama, al estar expuesta a una situación traumática y estresante, han adquirido la capacidad de resistir al dolor y obtener los aspectos positivos de dicha experiencia para salir más fortalecidas, lo cual redundará en un mayor nivel de bienestar subjetivo 0 satisfacción con la vida.

\section{OBJETIVOS DE LA INVESTIGACIÓN}

El ánimo del presente trabajo surge de la inquietud por profundizar en el conocimiento del bienestar de mujeres con cáncer de mama y la posible relación con aspectos psicosociales como la resiliencia.

Por ello, los objetivos específicos que se contemplan en el presente estudio son los siguientes:

Conocer los niveles de resiliencia en mujeres con diagnostico de cáncer de mama.

Estudiar el nivel de bienestar subjetivo o satisfacción con la vida en mujeres con cáncer de mama.

Analizar la relación entre los niveles de resiliencia y satisfacción con la vida en esta población.

Analizar la posible capacidad explicativa y/o predictiva de los niveles de resiliencia, en cuanto a los de satisfacción con la vida en este colectivo.

\section{MUESTRA Y/O PARTICIPANTES}

Al objeto de poder llevar a cabo el presente estudio, se ha seleccionado una muestra formada por 30 mujeres con diagnóstico de cáncer de mama que estaban siendo atendidas en la Unidad de Oncología del Hospital General de Jerez de la Frontera y en el Hospital Puerta del Mar de Cádiz en el momento de la evaluación. La media de edad fue de 47,47 años (33-59), con una DT=6.356. Respecto al tiempo medio transcurrido desde el diagnóstico de la enfermedad hasta el momento de la evaluación es de 9,9 meses (DT= 8,541).

En cuanto al estado civil encontramos que el $10 \%$ de la muestra eran solteras, el $66.7 \%$ estaban casadas, el $10 \%$ divorciadas, el 6,7\% eran pareja de hecho y el 6,7 eran viudas.

Respecto al nivel de estudios, hallamos que el 3,3\% no tenía estudio, el 20\% poseían estudios primarios, el $36,7 \%$ estudios secundarios (formación profesional y bachillerato) y el $40 \%$ estudios superiores.

Referente a la situación laboral, el 40\% estaban en situación activa, el 23,3\% se encontraban en situación de desempleo, el 23,3\% eran pensionistas y por último el 13,3\% estaban en otras situaciones laborales, como prácticas en empresa o trabajos no regularizados legalmente.

Por último, respecto a los datos médicos indicar que el $56,7 \%$ de las mujeres habían recibido como tratamiento la cirugía radical o mastectomía, todas estaban recibiendo tratamiento de quimioterapia y el $3,3 \%$ se encontraba en el estadio 0 de la enfermedad, el $13,3 \%$ en el estadio 1 , el $56,7 \%$ en el estadio 2 , el $23,3 \%$ en el estadio 3 y el $3,3 \%$ en el estadio 4 , correspondiendo con una paciente que presentaba metástasis en el hígado.

\section{METODOLOGÍA Y/O INSTRUMENTOS UTILIZADOS}

Los criterios de inclusión que se tuvieron en cuenta para seleccionar la muestra fueron: ser 
mujer con diagnóstico de cáncer de mama, estar en proceso de tratamiento activo de la enfermedad, no estar diagnosticada de otras patologías graves concomitantes al proceso oncológico, tener una edad comprendida entre los 20 y 60 años, con un nivel de lectura y escritura que posibilitara la comprensión de las pruebas que se le presentasen, no encontrarse en el momento del estudio en tratamiento psiquiátrico y/o psicológico alguno debido a un trastorno mental grave, no estar en el momento de la entrevista bajo medicación psicoactiva y no presentar ninguna patología grave 0 incapacitante.

La selección de las participantes que conformaron el total de la muestra se realizó de forma aleatoria atendiendo a su presencia o llegada a la Unidad de Oncología del Hospital General de Jerez de la Frontera, y del Hospital Universitario Puerta del Mar, situado en la localidad de Cádiz.

El procedimiento llevado a cabo para el acceso a las mujeres en tratamiento adyuvante por cáncer de mama, se procedió de dos modos. Uno, a través del personal de enfermería quien, mientras atendía a las pacientes en la sala de tratamiento, les mostraba el Consentimiento informado para su aprobación y firma. Posteriormente, una vez el evaluador disponía de los datos de la paciente, se contactaba con ella, vía telefónica, y se concertaba una cita en las instalaciones cedidas por el Hospital. Otro modo era acceder a las pacientes personándose el evaluador en la sala de tratamientos del Hospital de Día de ambos centros, y seleccionando directamente a las mujeres diagnosticadas de cáncer de mama que se encontraban presentes en ese momento, se les informaba del estudio y si prestaban su consentimiento se iniciaba la evaluación.

El total de la entrevista tuvo una duración de una hora aproximadamente. Las pruebas fueron autoadministradas, no obstante, el evaluador estaba presente en todo momento para la aclaración de cualquier duda que pudiera surgir a las pacientes.

La información obtenida fue introducida y analizada en el programa informático SPSS versión 22.0.

Para completar la evaluación se administraron los siguientes instrumentos:

Cuestionario de elaboración propia: donde se recogieron datos sociodemográficos, como la edad, estado civil, nivel de estudios, situación laboral, si padecían o no trastorno psicológico o psiquiátrico, o si estaban o habían estado en tratamiento psiquiátrico o psicológico, así como se recogieron datos de salud como el tiempo que llevaban con la enfermedad, el tipo de tratamiento que estaban recibiendo, o el estadio en el que había sido diagnosticada la misma.

Para la evaluación de la resiliencia se administró la Escala de Resiliencia de Wagnild \& Young (1993), en su adaptación al castellano por Novella (2002). El cuestionario se compone de 25 ítems evaluados mediante una escala de 7 puntos, donde 1 es en desacuerdo, y un máximo de acuerdo es 7. Los participantes han de indicar el grado de conformidad con el ítem, ya que todos son calificados positivamente, el rango de puntaje varía entre 25 y 175 (Wagnild \& Young, 1993). A partir de 75 es indicativo de unos niveles adecuados de resiliencia, teniéndose en cuenta el siguiente baremo:

0-74: No resiliencia

74-100: Resiliencia baja

100-125: Resiliencia media

125-150: Resiliencia alta

150-175: Resiliencia muy alta

Además de proporcionar una medida global de Resiliencia, aporta información de 5 dimensiones que forman parte de la misma, como son:

Ecuanimidad: un ejemplo de ítem sería: "Rara vez me pregunto cuál es la finalidad de todo", en el presente trabajo esta dimensión presenta una fiabilidad de $\alpha$ de Crobach $=0,53$. 
Perseverancia: un ejemplo de ítems sería "Cuando planeo algo lo realizo", en la presente investigación muestra una fiabilidad de $\alpha$ de Cronbach=0,91.

Confianza en sí mismo: por ejemplo, "Me siento orgulloso de haber logrado cosas en mi vida", la fiabilidad de esta dimensión en este estudio es de $\alpha$ de Cronbach: 0,82.

Satisfacción Personal: por ejemplo, "Por lo general encuentro algo de lo que reírme", en esta dimensión la fiabilidad es de $\alpha$ de Cronbach: 0,38. Dado el bajo índice de fiabilidad, esta dimensión no fue tenida en cuenta para los análisis de los datos.

Sentirse bien Solo: por ejemplo, "Puedo estar solo si tengo que hacerlo", esta dimensión presenta una fiabilidad de $\alpha$ de Cronbach: 0,57.

La puntuación de Resiliencia Total, en el presente trabajo presentó una fiabilidad de $\alpha$ de Cronbach: 0,91 .

Como se ha indicado anteriormente, para el análisis de los resultados obtenidos en esta prueba, no se ha podido tener en cuenta la dimensión "Satisfacción Personal" dado que presentaba un índice de fiabilidad no aceptable estadísticamente.

En estudios llevados a cabo por Novella (2002) se demostró la validez concurrente por altos índices de correlación con mediciones bien establecidas de constructos vinculados con la resiliencia.

Para la evaluación del bienestar subjetivo o satisfacción con la vida se utilizó el Satisfaction With Life Scale (SWLS).

La SWLS creada por Diener, Emmons, Larsen \& Griffin (1985) evalúa los juicios subjetivos sobre la satisfacción de la vida de un sujeto en función de las comparaciones que hace éste entre las circunstancias de su vida y un estándar, autoimpuesto, que considera adecuado. Consta de cinco ítems que evalúa el juicio global que hacen las personas sobre la satisfacción con su vida, valorada con una escala tipo Likert de 1 (muy en desacuerdo) a 7 (muy de acuerdo). La puntuación de la escala oscila entre 5 y 35.

Las puntuaciones del SWLS son interpretadas en términos absolutos (Diener et al., 1985). Así una puntuación de 20 representa el punto de corte en la escala y correspondería con la puntuación en la que el sujeto está igualmente satisfecho que insatisfecho con su vida.

Puntuaciones de 5-9 muy insatisfecho con la vida.

Puntuaciones de 10-14 insatisfecho con la vida.

Puntuaciones de 15-19 ligeramente insatisfecho con la vida.

Puntuaciones de 21-25 ligeramente satisfecho con la vida.

Puntuaciones de 26-30 satisfecho con la vida.

Puntuaciones de 31-35 muy satisfecho con la vida.

La SWLS ha mostrado una alta consistencia interna con unos alpha de Cronbach que oscilan entre 0.89 y 0.79 . Las correlaciones ítem-total oscilan entre 0.80 y 0.51 (Pavot \& Diener, 1993). Respecto a la fiabilidad test-retest se han encontrado índices que se encuentran entre 0.83 para periodos de dos meses y 0.50 para periodos más largos (Pavot \& Diener, 1993).

En esta investigación la escala ha presentado un $\alpha$ de Cronbach $=0.82$.

\section{RESULTADOS}

Al fin de dar respuesta al primer objetivo se calculó la media de la puntuación directa obtenida en la escala de resiliencia, la cual es de 144,63 que se corresponde, según el baremo de la prueba, con un nivel alto de resiliencia, dado que se encuentra dentro del intervalo 125-150. A la luz de estos resultados se puede inferir que las mujeres con cáncer de mama que forman parte de este estudio han desarrollado la capacidad de resistir al dolor y sufrimiento producido por la enfermedad, saliendo fortalecida de la misma. 
En cuanto al segundo objetivo, se procedió de igual forma a calcular la media de la puntuación directa que habían obtenido en la Escala de Satisfacción con la Vida, siendo ésta de 28,36. Atendiendo al baremo de la escala, esta puntuación señala que las participantes se muestran satisfechas con su vida, siendo esto un indicativo de elevados niveles de bienestar subjetivo.

Para responder al objetivo tres, se llevó a cabo un análisis de correlación bivariada entre las variables Resiliencia (y las dimensiones que la componen) y la Satisfacción con la vida.

Tabla 1.

Correlaciones bivariadas entre la Resiliencia y las distintas dimensiones de ésta y la Satisfacción con la vida

\begin{tabular}{lc}
\hline Resiliencia y subescalas & $\begin{array}{c}\text { Satisfacción con la } \\
\text { vida }\end{array}$ \\
\hline Resiliencia &, $663^{* *}$ \\
Ecuanimidad &, $562^{* *}$ \\
Confianza en sí mismo &, $677^{* *}$ \\
Perseverancia &, $576^{* *}$ \\
\hline
\end{tabular}

Como se puede observar en la Tabla 1, tanto la variable Resiliencia globalmente considerada, como las dimensiones Ecuanimidad, Confianza en sí mismo y Perseverancia muestran una correlación positiva estadísticamente significativa con el nivel de bienestar subjetivo o satisfacción con la vida.

Por último, para dar respuesta al cuarto objetivo y comprobar si las correlaciones encontradas implican, a su vez que la Resiliencia (con sus diferentes dimensiones) explican y predicen los niveles de Satisfacción con la vida en esta muestra, se realizó un análisis de regresión lineal, tomándose como variable dependiente la Satisfacción con la vida y como variable predictora la Resiliencia (con sus diferentes dimensiones) cuyos resultados se muestran en la tabla 2.

Tabla 2. Resumen del análisis de regresión efectuado

\begin{tabular}{|c|c|c|c|}
\hline Variable /variable predictor & $\% R^{2}$ & $F$ & $\mathrm{~B}$ \\
\hline Satisfacción con la vida & $56 \%$ & $(5,24) 6,115$ (sig.001) & \\
\hline • Resiliencia & & &, $659(\operatorname{sig}, 568)$ \\
\hline - Ecuanimidad & & &,$- 358(\operatorname{sig}, 721)$ \\
\hline - Confianza en sí mismo & & & ,378 (sig, 441) \\
\hline - Perseverancia & & &,$- 186(\operatorname{sig}, 737)$ \\
\hline
\end{tabular}

La satisfacción con la vida es explicada en un $56 \%$ por la variable Resiliencia, compuesta por las dimensiones Ecuanimidad, Confianza en sí mismo y Perseverancia. Sin embargo, tal y como se refleja en la tabla 2, ninguna de las dimensiones de la resiliencia muestra una capacidad predictiva significativa respecto de la satisfacción con la vida. Únicamente se puede observar que la resiliencia globalmente considerada muestra una significación de 0,568, por lo que a pesar de estar cerca de ser significativa, no se puede afirmar que así lo sea.

Por lo que estos resultados han permitido conocer la relación existente entre la Resiliencia y los niveles de Satisfacción con la vida o bienestar subjetivo, sin embargo no ha permitido afinar más los datos para conocer qué dimensión de la primera explica y predice los niveles de la segunda, datos que hubieran sido de relevante utilidad de cara a plantear programas de intervención psicológica con mujeres con cáncer de mama. 


\section{DISCUSIÓN}

Los resultados obtenidos arrojan la existencia de elevados niveles de resiliencia en las mujeres con diagnóstico de cáncer de mama que han sido evaluadas para este estudio. Eso quiere decir que el hecho de estar experimentando un acontecimiento traumático y adverso como es el padecimiento de cáncer de mama, con la incertidumbre del pronóstico de la enfermedad, los efectos secundarios que los tratamientos médicos tienen sobre la salud física y psicológica de estas mujeres, así como todos los cambios en su vida cotidiana derivados del diagnostico, han activado un mecanismo de crecimiento postraumático. Es decir, a pesar del sufrimiento, estas mujeres son capaces de obtener aspectos positivos de la enfermedad, y salir fortalecidas de dicha experiencia. Estos datos van en consonancia con lo aportado por García-Serrablo, Acevedo y Ortiz (2011) quienes indican que las mujeres con cáncer de mama han llegado a tener una percepción de su enfermedad de forma positiva, encontrándole beneficios a la misma, después de haber pasado por el sufrimiento derivado de los tratamientos médicos y la incertidumbre sobre el pronóstico. También Crespo y Rivera (2012) hallaron que mujeres con cáncer de mama mencionaban haber salido fortalecidas tras la enfermedad, pasaron a tener más fe, sentirse más capaces de solucionar problemas, poder relacionarse mejor con las personas que le rodean y tener menor preocupación sobre la enfermedad, es decir, con elevados niveles de resiliencia.

Por último, en cuanto a la relación entre la Resiliencia y la Satisfacción con la vida, los resultados arrojan relaciones positivas entre ambas variables, es decir, cuanto mayores niveles de resiliencia mayores niveles de bienestar subjetivo, lo cual va en la misma línea de los resultados hallados por Bellizzi y Blank (2006), Scrignaro, Barni y Magrin (2010), Vázquez y Castilla (2007), Aspinwall y MacNamara (2005), Aydin, (2008), Bozo et al., (2009), Salsman, Segerstrom, Brechting, Carlson y Andykowski (2009), quienes indicaban que las mujeres con cáncer de mama que presentaban mayores niveles de resistencia a la adversidad, y salían fortalecidas de la misma, presentaban mejores niveles de bienestar.

Si bien, no se puede confirmar que la resiliencia sea un predictor de la satisfacción con la vida o bienestar subjetivo en las mujeres con cáncer de mama que participaron en este estudio. Esto puede ser debido a varios factores entre los que cabe destacar el pequeño tamaño de la muestra. Por lo que de cara a futuros estudios, se considera crucial aumentar el tamaño de la muestra para poder afinar más en los resultados y aumentar el conocimiento de la dirección de las relaciones encontradas entre las dos variables principales de este trabajo.

\section{CONCLUSIONES}

A la luz de los resultados obtenidos mediante el presente estudio se puede concluir que:

Las mujeres con diagnóstico de cáncer de mama que participaron en este estudio muestran elevados niveles de resiliencia.

Las mujeres que forman parte de esta muestra están muy satisfechas con su vida, con elevados niveles de bienestar subjetivo.

La Resiliencia, tanto globalmente considerada como en sus dimensiones Ecuanimidad, Confianza en sí mismo y Perseverancia guarda una relación positiva estadísticamente significativa con la satisfacción con la vida.

En la muestra estudiada no se ha hallado que la resiliencia sea un predictor de elevados niveles de satisfacción con la vida o bienestar subjetivo.

\section{REFERENCIAS BIBLIOGRÁFICAS}

Aguila, M. (2000). Diferencia en la resiliencia según género y nivel socioeconómico en adolescentes. Lima: Universidad Nacional de Federico Villareal. 
Alonso Fernández, C. \& Bastos Flores, A. (2011). Intervención psicológica en pacientes con cáncer. Clínica Contemporánea, 2(2), 187-207.

Aspinwall L.G. \& MacNamara A. (2005). Taking positive changes seriously. Cancer, 1(104), 25492556.

Atienza, F.L., Pons, D., Balaguer, I. \& García-Merita, M. (2000). Propiedades psicométricas de la escala de satisfacción con la vida en adolescentes. Psicothema, 12(2), 314-319.

Aydin, E. (2008). Trauma and Resilience in Women Diagnosed with Breast Cancer: A Transactional Analysis Perspective. Transactional Analysis Journal, 38(4), 323-334.

Barreto, P. \& Soler, C. (2008). Predictores del duelo complicado. Psicooncología, 5(2-3), 383-400.

Bellizzi, K.M. \& Blank, T.0. (2006). Predicting Posttraumatic Growth in Breast Cancer Survivors. Health Psychology, 25(1), 47-56.

Bernardi, D., Errante, D., Galligioni, E., Crivellari, D., Bianco,A., Salvagno, L. \& Fentiman, I. S. (2008). Treatment ofbreast cancer in older women. Acta Oncologica, 47,187-198.

Bozo, O., Gündogdu, E. \& Büyükasik-Colak, C. (2009). The moderating role of different sources of perceived social support on the dispositional optimism- posttraumatic growth relationship in postoperative breast cancer patients. Journal Health Psychology 14, 1009-1020.

Crespo, L. \& Rivera, M.L. (2012). El poder de la resiliencia generado por el cáncer de mama en mujeres de Puerto rico. Revista Puertorriqueña de Psicología, 23, 109-126.

Diener, E., Emmons, R., Larsen, R. J. \& Griffin, S. (1985). The Satisfaction With Life Scale. Journal of Personality Assessment, 49(1), 71-75.

Diener, E., Scollon, C.N. \& Lucas, R.E. (2009). The evolving concept of subjetive well-being. En Diener, E. (Ed.) Assesing well-being. The collected works of Ed Diener. (pp.67-100). New York: Springer.

García-Serrablo, P., Acevedo, S. \& Ortiz, N. (2011). Benefit finding in breast cancer patients: The perspective of Puerto Rican women, 118th APA Convention, Washington: DC.

Guil, R., Zayas, A., Gil-Olarte, P., Guerrero, C. \& Mestre, J. M. (2017). Ejercicio físico y aspectos psicosociales y emocionales en mujeres con cáncer de mama. International Journal of Developmental and Educational Psychology. Revista INFAD de Psicología., 4(1), 327-338.

Guil, R., Zayas, A., Gil-Olarte, P., Guerrero, C., González, S. \& Mestre, J. M. (2016). Bienestar psicológico, optimismo y resiliencia en mujeres con cáncer de mama. Psicooncología, 13(1), 127.

Novella, A. (2002). Incremento de la resiliencia luego de la aplicación de un programa de psicoterapia breve en madres adolescentes. Tesis para optar el grado de Magíster en Psicología, mención en Psicología clínica y de la salud. Universidad Nacional Mayor de San Marcos, Lima, Perú.

Pavot, W. \& Diener, E. (1993). The affetive and cognitive contest of self reports measures of subjetive well-being. Social Indicators Research, 28(1), 1-20.

Salsman, J.M., Sergerstrom, S.C., Brechting, E.H., Carlson, C.R. \& Andrykowski, M.A. (2009). Posttraumatic growth and PTSD symptomatology among colorectal cancer survivors: a 3-moth longitudinal examination of cognitive processing. Psychooncology, 18 (1), 30-41.

Scrignaro, M., Barni, S. \& Magrin, M.E. (2010). The combined contribution of social support and coping strategies in predicting post-traumatic growth: a longitudinal study on cancer patients. Psychooncology, 20(8), 823-831.

Sociedad Española de Oncología Médica (SEOM) (2016). Las Cifras del Cáncer en España 2014. Madrid.

Tescari, G. (2012). Resiliencia y acontecimietos vitales estresantes en pacientes con cáncer de mama y pacientes con linfoma. Universidad de los Andes.

Vázquez, C. \& Castilla, C. (2007). Emociones positivas y crecimiento postraumático en el cáncer de 
mama. Psicooncología, 4(2-3), 385-404.

Vázquez, C., Duque, A. \& Hervás, C. (2013). Escala de Satisfacción con la Vida (SWLS) en una muestra representativa de españoles adultos: Validación y datos normativos. Spanish Journal of Psychology, 1-37.

Vera-Villarroel, P., Urzúa, A., Pavez, P., Celis-Atenas, K. \& Silva, J. (2012). Evaluation of subjetive well-being: Analysis of the Satisfaction with Life Scale in Chilean population. Universitas Psychologica, 11, 719-727.

Von Humboldt, S., Carneiro, F. \& Leal, I. (2017). Más allá de cáncer de mama en la edad avanzada: bienestar subjetivo en mujeres mayores con cáncer de mama en remisión. Revista Argentina de Clínica Psicológica, 26(2).

Wagnild, G. \& Young, H. (1993). Development and psychometric evaluation of the resilience scale. Journal of Nursing Measurement, 1(2), 165-167.

Zayas, A. \& Guil, R. (2018). El cáncer de mama desde un enfoque biopsicosocial de la salud. Perspectiva salutogénica. Sevilla: Fenix Editora. 